\title{
Management of Teeth with Bilateral Radix Entomolaris: A Case Report
}

\author{
Amulya Vanti ${ }^{1 *}$, Madhu Pujar ${ }^{2}$, Sheetal Ghivari ${ }^{3}$, Atul Chandra.M ${ }^{4}$, Chandra Shekhar \\ Bynagari ${ }^{5}$, Dr Pallavi Gopeshetti \\ Department of Conservative Dentistry and Endodontics, Maratha Mandal, S Nathajirao G Halgekar Institute of \\ Dental Sciences and Research Centre Belgaum
}

*Corresponding Author: Amulya Vanti, Department of Conservative Dentistry and Endodontics, Maratha Mandal, S Nathajirao G Halgekar Institute of Dental Sciences and Research Centre Belgaum

\begin{abstract}
Understanding of the presence of an additional root and its root canal anatomy is essential for successful treatment outcome. Usually mandibular molars have two roots with three canals (mesiobucccal, mesiolingual \& distal) but in few teeth, the number of roots and canals vary. mandibular molars which is associated with an extra root on the lingual side known as Radix Entomolaris,

This case report discuss the prevalence, morphology, clinical diagnosis, problems encountered during treatment and prognosis of tooth in bilateral radix Entomolaris case.
\end{abstract}

Keywords: Bilateral Radix Entomolaris, Anatomic root variation

\section{INTRODUCTION}

According to Weine, the main causes of endodontic failure are improper identification of canals, untreated major canals; incorrect canal instrumentation, incomplete obturation.

And Magnification, radiographs, operating microscope, loupes and illumination are aids that must be utilized to achieve this goal [1].

Failure to recognize the presence of an additional canal may result in unsuccessful treatment and may be the origin of acute flare ups during and after treatment [2].

Radix Entomolaris is a developmental variation commonly found in mandibular molars which is associated with an extra root on the lingual side known as Radix Entomolaris [3],

Incidence of Bilateral Radix Entomolaris is reported to vary from 37.14 to $67 \%$.

Here we discuss the bilateral management of radix entomolaris of mandibular first molar teeth [4].

\section{CASe Report 1}

A 35 year old female patient reported to Department of Conservative Dentistry \& Endodontics with a chief complaint of severe pain in the lower left and right back tooth region since one week.
Pain was of sharp, throbbing, continuous in nature, aggravated on mastication with 36 and 46.

A diagnostic radiograph was taken it showed radiolucency involving pulp and periodontal ligament widening seen.

On observation of radiograph there appears to be an additional root bilaterally.

Another radiograph has been taking with mesial and distal angulations.

Local anesthesia was administered and the tooth was isolated under rubber dam. Access cavity preparation was modified from a triangular to a trapezoidal form for proper accessibility of all the canals.

Working length was determined radigraphically. Cleaning and shaping of canals was performed with protaper rotary instruments in a step-down manner and obturation was done with cold lateral condensation with 36 and 46.

Glyde lubricant was used and the canals were irrigated using sodium hypochlorite and normal saline and final irrigation was done with chlorhexidine. Post obturation restoration was restored with composite.

Follow-up was done for three months and no signs and symptoms were noticed. 


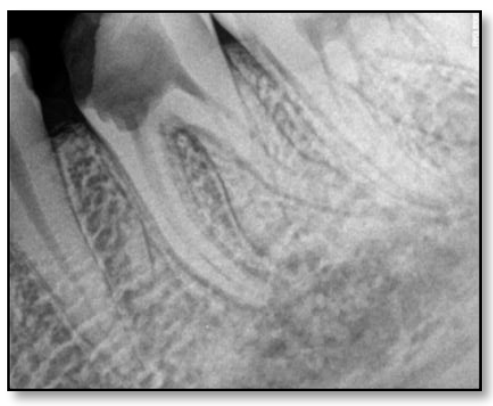

Figure 1 A

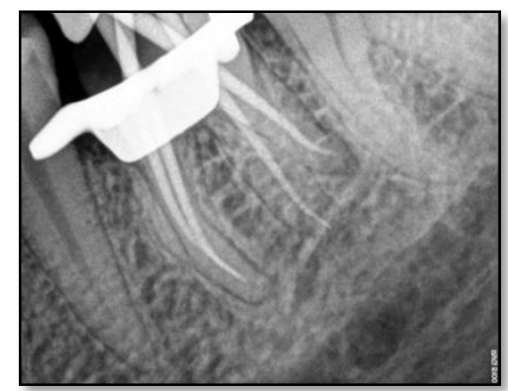

Figure 1 B

Figure 1. Figures $1 a \& 1 b$ shows Pre-operative and Post-obturation radiographs showing all the four canals 36

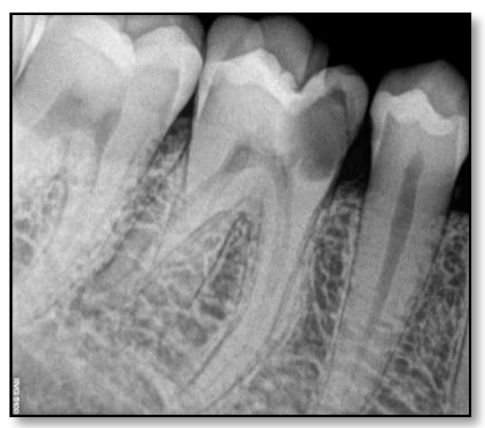

Figure 2 A

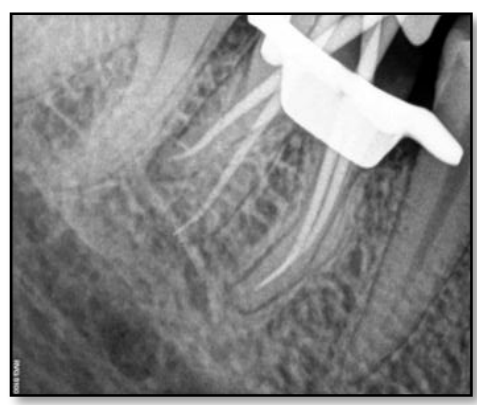

Figure $2 B$

Figure2. $2 a$ \& $2 b$ Shows Pre-Operative Working Length and Post-Obturation Radiographs Showing all the Four Canals 46

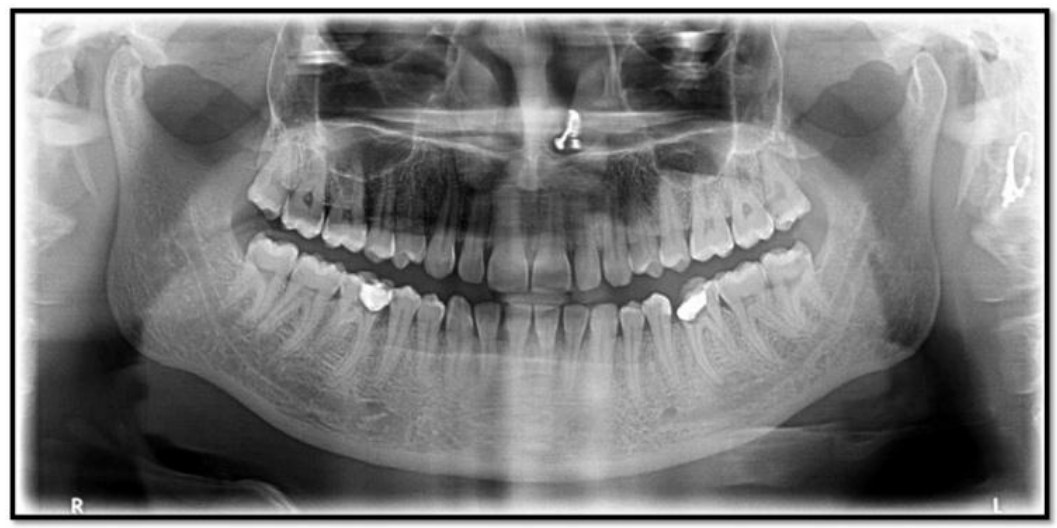

Figure3. Preoperative OPG Showing Bilateral Radix Entomolaris

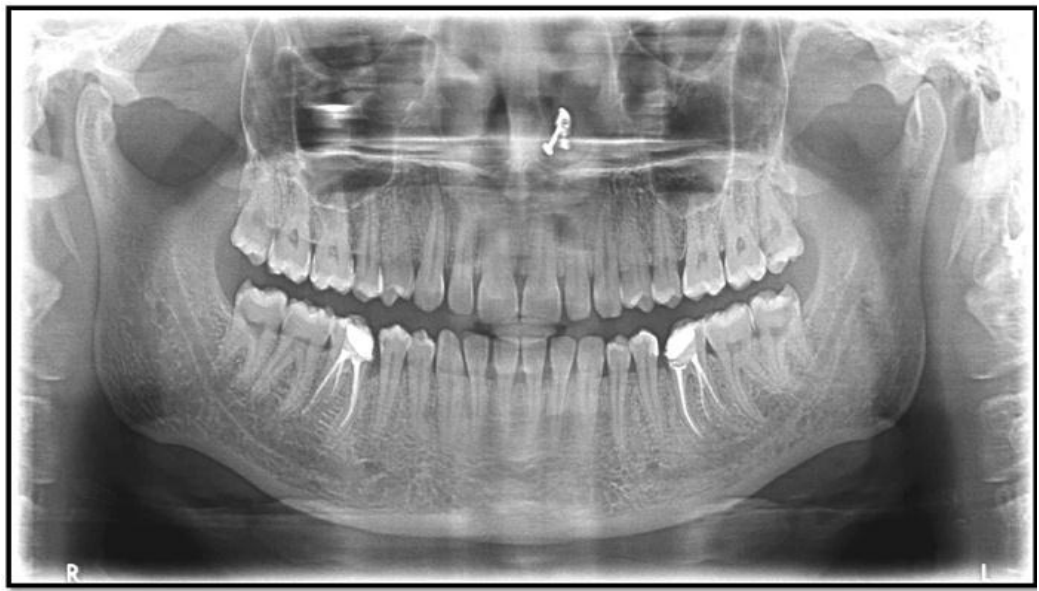

Figure4. Post Obturation OPG Showing Bilateral Radix Entomolaris 


\section{DISCUSSION}

Radix Entomolaris (RE) was first described by Carabelli. It is characterized by the presence of an additional or extra third root, which is typically found disto-lingually [5].

Radix entomolaris can be found in the first, second, and third mandibular molars, Highest among the population of Mongolian origin. Radix Entomolaris is not very common in African, Eurasian, and Caucasian. Prevalence of RE, with a range from2. 19-13.3\%, among the Indian population.

There is no significant difference was found in the prevalence of $\mathrm{RE}$ according to gender ${ }^{6}$. Similarly no difference was found in the side of occurrence. The bilateral occurrence of RE is reported to vary from 37.14 to $67 \%$.

Frequently for a correct diagnosis minimum of two diagnostic radiographs are necessary using buccal object rule. Even the presence of an extra cusp may sometimes indicate the presence of radix entomolaris $[7,8]$.

Access cavity preparation should be modified usually from a triangular to a trapezoidal shape. The modification should be done following the dentinal map [9].

Advanced diagnostic aids help in the better identification and visualization of all the canals.

The diagnosis and management of RE are of paramount importance from the point of endodontic success $[10,11]$.

\section{CONClusion}

The knowledge of variation in anatomy of mandibular permanent first molars, Proper interpretation of radiographs.

And identification of number of roots and morphology and proper cleaning and shaping of the canals and three dimensional obturation is very important in success of endodontic treatment.

\section{REFERENCES}

[1] Fabra-Campos H (1989) Three canals in the mesial root of mandibular first permanent molars: a clinical study.IntEndod J 22: 39-43.

[2] Curzon ME, Curzon JA (1971) Three-rooted mandibular molars in the Keewatin Eskimo.J Can Dent Assoc (Tor) 37: 71-72.

[3] Turner CG 2nd (1971) Three-rooted mandibular first permanent molars and the question of American Indian origins.Am J PhysAnthropol 34: 229-241.

[4] De Moor RJ, Deroose CA, Calberson FL (2004) The radix entomolaris in mandibular first molars: an endodontic challenge. IntEndod J 37: 789-799.

[5] Bolk L (1914) WelcherGebibreihegehoren die Molaren an? Z MorpholAnthropol 17: 83-116.

[6] Abella F, Patel S, Durán-Sindreu F, Mercadé M, Roig M (2012) Mandibular first molars with disto-lingual roots: review and clinical manage ment. IntEndod J 45: 963-978.

[7] Song JS, Choi HJ, Jung IY, Jung HS, Kim SO (2010) The prevalence and morphologic classif ication of distolingual roots in the mandibular molars in a Korean population.J Endod 36: 653657.

[8] Reichart PA, Metah D (1981) Three-rooted permanent mandibular first molars in the Thai. Community Dent Oral Epidemiol 9:191-19 2.

[9] Walker RT, Quackenbush LE (1985) Threerooted lower first permanent molars in Hong Kong Chinese.Br Dent J 159: 298-299.

[10] Ingle JI, Heithersay, GS, Hatwell GR (2002) Endodontic Diagnostic Procedures. BC Decker, London, UK.

[11] Gopikrishna V, Reuben, J., Kandaswamy, D (2008) Endodontic management of a maxillary first molar with two palatal roots and a single fused buccal root diagnosed with spiral compu ted tomography-a case report. Oral Surgery Oral Medicine, Oral Pathology, Oral Radiology and Endodontology 105:e74-e8.

Citation: Amulya Vanti, et al. Management of Teeth with Bilateral Radix Entomolaris: A Case Report. ARC Journal of Dental science. 2019; 4(3):13-15. doi:dx.doi.org/10.20431/2456-0030.0403003.

Copyright: (1) 2019 Authors. This is an open-access article distributed under the terms of the Creative Commons Attribution License, which permits unrestricted use, distribution, and reproduction in any medium, provided the original author and source are credited. 